\title{
L'intégration des concepts de résilience dans le domaine de la sécurité alimentaire : regards croisés
}

\author{
Simon Vonthron ${ }^{1, *}$, Sandrine Dury ${ }^{1}$, Abigail Fallot ${ }^{2}$, Arlène Alpha ${ }^{1}$ et François Bousquet ${ }^{2}$ \\ ${ }^{1}$ Cirad, UMR Moisa, TA C-99/15, 73, rue J.F.-Breton, 34398 Montpellier cedex 5, France \\ 2 Cirad, UMR GREEN, TA C-47/F, Campus international de Baillarguet, 34398 Montpellier cedex 5, France
}

\begin{abstract}
Résumé - On observe depuis les années 2000 la volonté des acteurs de la sécurité alimentaire de rapprocher résilience et sécurité alimentaire. Ce rapprochement n'apparaît cependant pas si évident au vu de la diversité d'éléments de langages utilisés pour les associer. Cet article vise donc à faire un état des lieux de l'association de ces notions, voire de leur intégration mutuelle. L'application de la résilience à la sécurité alimentaire metelle en avant les mêmes dimensions que lorsqu'elle est appliquée aux systèmes socioécologiques ? Y aurait-il ainsi plusieurs concepts de résilience ? Quels sont les apports en termes programmatique et analytique dans le champ de la sécurité alimentaire ? Quels difficultés ou défis posent cette intégration ? Une analyse de la littérature a été menée à partir de références scientifiques ainsi que de documents de cadrage, de projets et programmes produits par des institutions, bailleurs de fonds et ONG. Deux enjeux se dégagent de ce rapprochement : la mesure de la résilience et les apports de cette approche en comparaison d'autres telles que la gestion des risques et la réduction de la vulnérabilité. Par ailleurs, alors qu'elle n'a pas de portée normative concernant les trajectoires des systèmes socioécologiques, la résilience est intrinsèquement considérée comme positive dans le domaine de la sécurité alimentaire. Cette acception de la résilience soulève alors des problèmes politiques de définition de ce qu'est une trajectoire favorable. L'accent mis, avec la résilience, sur les trajectoires du système, permet toutefois de relier court terme et long terme, tant pour comprendre les perturbations que pour concevoir des interventions. Enfin, les caractères holistique et multiscalaire de la résilience se retrouvent dans l'ensemble de la bibliographie étudiée, constituant une " avancée » dans le domaine de la sécurité alimentaire.
\end{abstract}

Mots clés : résilience / sécurité alimentaire / système socioécologique / aide humanitaire / développement

Abstract - An integrated perspective on resilience and food security. Since the beginning of the 2000 s, resilience and food security have become associated in literature. However, the diversity of terminology shows that this association is not so easy. This paper objective is to assess the state of the art concerning the different forms of associations between food security and resilience. Does using resilience in the food security field and in the social-ecological system field, highlight the same features? Therefore, do several resilience concepts exist? What does it bring to programs and analysis carried out into food security field? What difficulties and challenges does this integration cause? We carried out a review using scientific literature as well as policy, projects and programs documents from the United Nations, funding agencies, or NGOs. We raise two specific issues: the resilience metrics in the food security scope, and the contribution of resilience in comparison with other approaches such as risk management and vulnerability reduction. In addition, while resilience is not associated with a normative meaning in the social-ecological system literature, it is always associated with a positive notion in the domain of food security. This raises the politically sensitive question of who is defining a good trajectory. Resilience is also associated with the analysis of the system trajectories, which makes possible to link short-term and long-term. That allows to understand perturbations as well as to anticipate potential responses and design interventions. Finally, holistic and multi-scale characteristics of resilience are found in the whole studied literature and that seems to be an improvement in the food security field. However, the passage from theory to practice is still ongoing and complex.

Keywords: resilience / food security / social-ecological system / humanitarian aid / development

\footnotetext{
* Auteur de correspondance : simon.vonthron@acadis.org
} 


\section{Introduction}

Si les liens entre la "résilience » et la «sécurité alimentaire »ont déjà été soulignés dans les années 1990 (Maxwell et Smith, 1992), ce n'est que depuis quelques années, et en particulier depuis la présentation du rapport sur le droit à l'alimentation au Conseil des droits de l'homme de l'ONU par O. de Schutter en 2008, que ce rapprochement s'impose dans les agendas des politiques de développement international (WFP, 2011 ; UE, 2012 ; USAID, 2012 ; FAO, 2013)

La notion de résilience, utilisée dans plusieurs disciplines, physique, psychologie, écologie, et en matière de développement (Barrett et Constas, 2014), est désormais utilisée dans le domaine de la sécurité alimentaire, qui est, dans le même temps et indépendamment, remontée au sommet des agendas du développement. La mise en œuvre d'une approche « résilience » dans l'ensemble des actions qui visent l'amélioration de la sécurité alimentaire (de l'urgence et du développement) est compliquée pour tous les acteurs impliqués. Aussi bien dans le domaine de la résilience que dans celui de la sécurité alimentaire, pris isolément, les controverses sont vives sur les définitions, les mesures, les priorités et les façons d'agir. La difficulté du rapprochement entre les deux termes se reflète dans une terminologie hésitante : "résilience à la sécurité alimentaire et nutritionnelle » (ACF International, 2012); « résilience dans le cadre de la sécurité alimentaire » (MAE, 2014), « resilience for food security and nutrition » (FAO, 2006) ; " resilience to food insecurity »; " food security and resilience » (UE, 2012); « resilience for food and nutrition security » (Fan et al., 2014) ; " resilience of their livelihoods through food security » (WFP, 2011) ; "resilience and secure nutrition »(UNSCN, 2010).

Cette contribution, basée sur un dialogue entre chercheurs des deux domaines, de la résilience appliquée aux systèmes socioécologiques et de la sécurité alimentaire, a pour objectif de faire un état des lieux des enjeux de ce rapprochement, et des opportunités et des risques qu'il comporte.

\section{Méthode et contexte de l'étude}

\subsection{Matériel et méthode}

Suite à un stage de fin d'étude dans le cadre du projet Résilience des populations pauvres et très pauvres et sécurité alimentaire dans la boucle du Mouhoun (REPAM), qui a consisté à construire un état de référence sur la situation alimentaire et nutritionnelle dans les provinces du Mouhoun et du Nayala au Burkina Faso (Vonthron, 2014), nous sommes revenus en détail sur les concepts. Nous avons identifié et analysé différents textes concernant la définition de la résilience, et son utilisation dans le domaine de la sécurité alimentaire (Vonthron et al., 2015). Le choix des textes a porté sur des références théoriques et historiques de la résilience ainsi que sur des documents spécifiques sur la sécurité alimentaire et la résilience provenant de projets, programmes et cadres d'intervention publiés par des institutions, bailleurs de fonds et organisations non gouvernementales (ONG). Nous avons identifié la majorité des références scientifiques grâce à nos différents réseaux professionnels. Au-delà de celles-ci, nous avons effectué une recherche complémentaire sur internet
- Google, Googlescholar et Web of Science : recherche dans les titres dans « All databases » - avec différentes combinaisons des mots-clés suivants : « résilience », « sécurité alimentaire », « sécurité alimentaire et nutritionnelle », « projet », « programme » ainsi que les noms de certaines institutions onusiennes, $\mathrm{ONG}$ et bailleurs de fonds. Les mêmes mots-clés et combinaisons ont été utilisés, traduits, pour une recherche en anglais. Les références correspondant à l'utilisation de la résilience dans le domaine des systèmes socioécologiques et de la sécurité alimentaire ont été retenues, tandis qu'ont été exclues les références des champs trop éloignés comme la psychologie ainsi que celles trop spécifiques à un produit. Nous avons également exclu les références focalisées sur la résilience et la sécurité alimentaire urbaine ou globale. Nous n'avons pas retenu toutes les références, mais celles majeures (théoriques et historiques), celles concernant les projets en cours ou récents, et celles qui nous ont paru particulièrement illustratives et/ou qui apportent une idée nouvelle par rapport aux précédentes.

$\mathrm{Au}$ total, 64 références sont considérées : 14 articles scientifiques, quatre ouvrages scientifiques, six communications à des conférences et séminaires, 40 documents d'institutions internationales de développement (UE, FAO, ONU) et/ ou d'ONG (CARE, Gret, ACF...). Ces derniers comprennent des documents de cadrage et de positionnement d'organismes intervenant dans les domaines de l'aide humanitaire et du développement, des rapports scientifiques commandités par ces mêmes organismes, des documents de projets ou de recensements de projets, des communications sur les actions menées ainsi que différents guides et notes méthodologiques. L'analyse de cette littérature grise et scientifique permet de commencer par retracer l'histoire de l'intégration de la notion de résilience aux travaux et interventions pour la sécurité alimentaire.

\subsection{Les initiatives de recherche et de développement associant résilience et sécurité alimentaire}

En 2012, la Commission européenne a soutenu le lancement de deux initiatives, en Afrique de l'Ouest (AGIR) et dans la Corne de l'Afrique (SHARE), qui se sont traduites par le financement de projets de développement centrés sur les problématiques de sécurité alimentaire et de résilience. Entre autres, le programme PRORESI au Burkina Faso, avec des projets tels que le REPAM (porté par le Gret et la Fédération des professionnels agricoles du Burkina [FEPA-B]) ou bien l'« Action en faveur de la résilience » (Pro-Resilience Action [PRO-ACT]) qui couvre des interventions au Sénégal. Cette tendance au financement de projets et programmes sur ces thématiques se retrouvent chez l'ensemble des grands bailleurs de fonds comme USAID (2012), AFD (2015), DFID (2011), GIZ (2015), la Banque mondiale, Banque africaine de développement (BAD, 2015). La thématique de la résilience appliquée au champ de la sécurité alimentaire est également portée dans les plaidoyers et la communication de grandes ONG de développement et/ou d'urgence telles Action contre la faim (ACF International, 2012), le Gret (2014), Oxfam (Hillier et Castillo, 2013) ou Save the Children (2015).

En parallèle, une série de recherches spécifiquement consacrées à la résilience dans le domaine de la sécurité 
alimentaire s'est développée, dans le sillage des travaux initiés par Alinovi et al. (2009). Des échanges entre communautés «opérationnelle » et «académique » sont organisés et visibles, par exemple à travers les travaux du Food Security Information Network (FSIN, 2014a,b). La plupart de ces travaux scientifiques ont un lien explicite avec les interventions de développement ou d'urgence. Ils s'en nourrissent et affichent l'intention de contribuer à leur conception, leur mise en œuvre plus efficace et leur évaluation. Les frontières entre productions de type académique (articles publiés dans des revues scientifiques, communication à des conférences scientifiques) et les autres productions de type « institutionnel » ou « de communication » de bailleurs de fonds ou d'acteurs de développement (rapports, policy briefs, etc.) sont très floues. Les idées et les gens porteurs de ces questions circulent entre les mondes du développement international et de la recherche et il serait artificiel de les séparer/dissocier.

Dans le contexte de redevabilité des dépenses publiques du développement actuel, et étant donné les liens entre chercheurs et acteurs du développement dans ces domaines, il n'est pas surprenant de constater qu'un des enjeux principaux des recherches concerne les méthodes de caractérisation et de mesure. On le retrouve sur un grand nombre de supports (articles scientifiques, rapports institutionnels, document de communication), en particulier à travers des propositions de construction d'indicateurs. Il est communément admis que la résilience constitue une caractéristique latente, multidimensionnelle et non mesurable directement, tout particulièrement dans le domaine socioéconomique. Plusieurs cadres analytiques (Alinovi et al., 2009 ; Béné et al., 2012 ; USAID, 2012 ; Barrett et Constas, 2014 ; FSIN, 2014a,b), différents indicateurs des dimensions de la résilience (USAID, 2013) et différentes méthodes d'agrégation des dimensions (Alinovi et al., 2009 ; FSIN, 2014a) ont été développés.

Un second enjeu que l'on peut observer renvoie à des questionnements plus critiques sur l'utilité ou le caractère novateur de la notion de résilience par rapport à d'autres notions. Certains auteurs posent ainsi la question des apports de la résilience par rapport aux approches de gestion des risques et de réduction de la vulnérabilité (Béné et al., 2012). D’autres pensent que cette notion est essentiellement une nouvelle mode et n'apporte rien de nouveau aux différents outils du développement : pas de nouvelle compréhension des modes de vie des populations en insécurité alimentaire et pas de changement des rapports de force (Ancey et al., 2014a,b). Certains (Quenault, 2015) y voient un avatar de l'idéologie néo-libérale, qui trouverait dans ce concept l'occasion de théoriser le transfert de responsabilité de crises sur les individus, incapables de faire face à des chocs. L'engouement pour la notion de résilience, suivi de ces questionnements et critiques, invite à mieux comprendre ce qu'est ou n'est pas la résilience.

Il nous semble cependant que la littérature sur « résilience et sécurité alimentaire » ignore en grande partie les fondements et l'histoire du concept de résilience tel qu'il a émergé, puis s'est développé dans le domaine des systèmes écologiques et sociaux. C'est pourquoi, nous allons maintenant comparer les diverses acceptions de la notion de résilience selon que celle-ci est associée aux systèmes socioécologiques ou à la sécurité alimentaire, puis nous présenterons les traits de la résilience qui nous apparaissent être des apports réellement nouveaux et importants dans le domaine de la sécurité alimentaire ; enfin, nous soulignons les difficultés posées par le passage de la théorie à la pratique.

\section{La résilience, de l'écologie au développement, notion neutre versus positive : les risques de ce glissement sémantique}

Holling (1973) distingue deux formes de résilience : l' « engineering resilience », et l' « ecological resilience ». La première, directement issue de la physique des matériaux, qualifie la capacité d'un système à reprendre son état initial suite à une déformation. Elle se caractérise souvent par la résistance du système et par sa vitesse de retour à l'état initial. Elle suppose l'existence d'un état d'équilibre que l'écosystème devrait retrouver après une perturbation. La seconde s'intéresse aux changements d'états et aux seuils qui caractérisent le passage d'un état à l'autre. Elle est caractérisée par l'ampleur de la perturbation maximale que le système est capable d'absorber sans changer d'état (Holling, 1996). L'étude de la résilience est donc devenue l'étude des trajectoires d'un système et les conditions dans lesquelles il passe d'un état à un autre. Les développements de Holling, qui s'intéressait au début aux écosystèmes se sont ensuite étendus aux systèmes sociaux et écologiques (SSE). Un système socioécologique est l'« assemblage de systèmes complexes dans lesquels les hommes font partie de la nature » (Berkes et Folke, 1998). Il comprend des éléments humains et biologiques, ainsi que les interactions dynamiques entre acteurs et écosystème. Il se rapproche de la notion de « territoire » utilisé en géographie humaine (Arnauld de Sartre et al., 2015). Après de nombreuses années de travail, les chercheurs ont proposé une nouvelle définition de la résilience des SSE : «La résilience d'un système socioécologique est sa capacité à absorber les perturbations d'origine naturelle (un feu provoqué par la foudre, une sécheresse, etc.) ou humaine (une coupe forestière, la création d'un marché, une politique agricole, etc.) et à se réorganiser de façon à maintenir ses fonctions et sa structure ; en d'autres termes, c'est sa capacité à changer tout en gardant son identité ») (Mathevet et Bousquet, 2014).

La résilience, telle que définie dans la littérature des SSE, est donc une caractéristique du système et n'a pas de portée normative intrinsèque. Certains systèmes peuvent être bloqués dans des « situations de piège » (économique ou écologique) et être en même temps « résilients », c'est-à-dire qu'ils absorbent des perturbations et reviennent alors sur des trajectoires qui ne sont pas souhaitables (Frantzeskaki et al., 2014). Certains auteurs parlent alors, pour qualifier ces situations, de résilience négative ou "perverse resilience » (Phelan et al., 2013 ; Haider et al., 2014). C'est pourquoi, en 2004, des chercheurs ont introduit la notion de transformabilité, c'est-à-dire la capacité des acteurs d'un système à créer un nouveau système lorsque la trajectoire du premier n'est plus acceptable (Walker et al., 2004).

Par contre, dans le domaine de la sécurité alimentaire, la résilience est une notion toujours présentée comme une caractéristique positive : « building ou enhancing resilience » 
(FAO, 2006 ; de Schutter, 2008 ; Frankenberger et al., 2012a, b ; USAID, 2012 ; WFP, 2013 ; UNFPA, 2015), « améliorer la résilience » (ACF International, 2012; Gret, 2014), « renforcer la résilience » (FAO, 2012; Heijmans, 2013; BAD, 2015 ; AFD, 2015 ; GIZ, 2015). Le système ou l'individu est « résilient » si, et seulement si, il est dans une trajectoire favorable (ACF International, 2012 ; UE, 2012 ; Hillier et Castillo, 2013 ; FSIN, 2014b ; Lallau, 2014). Des situations défavorables persistantes, qui seraient qualifiées de résilientes dans un cadre d'analyse socioécologique (Haider et al., 2014), telles que les pièges à pauvreté, ne sont pas évoquées ou alors sont qualifiées de trappes de non-résilience (Lallau, 2011).

Remettre en cause cette conception positive de la résilience soulève alors un problème politique : comment décider si une trajectoire est favorable ou non, et qui en décide ? La réponse à de telles questions apparaît en effet subjective et dépendante de l'échelle d'analyse - ce qui est favorable à un individu ne l'est pas nécessairement pour un ménage et/ou l'ensemble d'une communauté. Béné et al. (2012) soulignent alors le risque suivant : un système peut être résilient (et un projet construit avec cet objectif, un succès) alors que ce système est inéquitable et ne résout pas les problèmes de vulnérabilité d'une partie de la population.

\section{Les apports potentiels de la résilience dans le domaine de la sécurité alimentaire}

Outre sa portée normative lorsqu'elle est appliquée à la sécurité alimentaire, la notion de résilience est aussi présentée comme une approche permettant d'articuler urgence et développement, et comme une approche systémique et multi-niveaux. Ces façons d'appréhender la lutte contre l'insécurité alimentaire ne sont pas nouvelles, mais l'utilisation du concept de résilience a été l'occasion d'une tentative de théorisation de ce qui pourrait assembler urgence et développement.

\subsection{Long terme et court terme : réconcilier développement et urgence}

Depuis de nombreuses années, et en particulier depuis la crise du Niger en 2005, de nombreux acteurs du développement et de l'urgence remettent en cause les instruments de lutte contre l'insécurité alimentaire et la séparation entre urgence et développement. En effet, de façon classique, trois grands types de politiques étaient mis en œuvre dans ce domaine : des politiques de long terme axées sur le développement agricole (et plus récemment sur la protection sociale), des politiques de prévention des crises et des politiques d'aide alimentaire d'urgence (Janin et Dury, 2012 ; Touzard et Temple, 2012). Un faisceau de causes explique cette remise en question :

- un besoin croissant de rationalisation des dépenses par les bailleurs de fonds et la volonté des organisations humanitaires d'aller vers la post-urgence ;

- l'émergence, dans le débat, des données de malnutrition qui mettent en évidence des taux très élevés de malnutrition dans des zones habituellement non suivies par les dispositifs de prévention des crises alimentaires ;
- le fait que les politiques et différents dispositifs dédiés à la surveillance et la prévention des crises n'ont su anticiper et prévenir ni la crise alimentaire de 2005 au Niger, ni la crise des prix internationaux de 2008.

De façon générale, l'objectif est alors de concevoir, comme le promeuvent Pingali et al. (2005) avec la « Twin-Track Approach », des interventions intégrant des réponses à court terme et des actions à long terme, ce qui est exactement l'ambition d'une approche par la résilience (ACF International, 2012 ; UE, 2012 ; USAID, 2012 ; Hillier et Castillo, 2013 ; Mathevet et Bousquet, 2014). L'articulation entre humanitaire et développement a depuis longtemps été recherchée (voir notamment le travail important de la Commission européenne pendant des années pour le « LRRD - Linking Rehabilitation, Relief and Development » [UE, 2001 ; VOICE et CONCORD, 2012]) mais a toujours eu du mal à se concrétiser, sans doute parce qu'au-delà des discours, sa mise en œuvre heurtait des intérêts, des habitudes et des institutions. Toujours est-il qu'à la fin des années 2000, redéfinir les relations entre le développement agricole (le long terme) et les crises alimentaires (le court terme) était devenu un enjeu scientifique et de développement (Crombé et Jézéquel, 2007 ; Michiels et Egg, 2007 ; Michiels et al., 2012).

D'un point de vue analytique, un des apports de la notion de résilience est de situer des événements plus ou moins brutaux par rapport à des trajectoires longues. Les trajectoires des individus/ménages, qui sont le niveau d'analyse privilégié des travaux en résilience et sécurité alimentaire, sont affectées par une multiplicité de perturbations modifiant leur niveau de bien-être ou de vie, tant dans le temps que dans leur diversité (inondations, épidémies, crise politique, etc.). La position du système sur la trajectoire informe alors des réponses possibles aux perturbations. À cet égard, la notion de résilience comme approche pour appréhender de façon approfondie les trajectoires peut être rapprochée de la notion de sécurisation, qui met elle aussi l'accent sur le temps long, sur les processus non linéaires, permettant aux individus/ménages d'atteindre l'état de sécurité alimentaire (Janin et Dury, 2012). Ceci étant, les documents étudiés rapprochent résilience et "état» de sécurité alimentaire plutôt que de faire référence à la sécurisation alimentaire. Par comparaison, une approche par la vulnérabilité permet de définir les états possibles de l'objet résultant de la relation perturbation-réponse mais n'analyse guère les conséquences sur les autres objets ni une trajectoire générale du système auquel appartient cet objet.

La notion de « perturbation » de la résilience correspondait parfaitement aux descriptions faites par plusieurs observateurs des « crises » et de l'insécurité alimentaire et nutritionnelle. Dans le cas du Niger par exemple (Michiels et al., 2012), l'accumulation et la répétition dans le temps des chocs (prix, climat, maladies, etc.) a conduit à l'augmentation de la vulnérabilité des individus, des ménages et des sociétés, et à la dégradation des capacités de réponse. En conséquence, selon une perspective de gestion des crises alimentaires, la résilience est mobilisée pour définir des actions de gestion destinées à faire évoluer le système vers un état désiré, alors que la vulnérabilité est plutôt employée pour des actions de gestion visant principalement à réduire les réponses négatives aux perturbations. 


\subsection{Une approche systémique et multi-niveaux}

La résilience dans les systèmes socioécologiques est holistique et multiscalaire (Resilience Alliance, 2010). Dans le domaine de la sécurité alimentaire également, de nombreux auteurs plaident pour une approche systémique et multiniveaux, voir, par exemple Touzard et Temple (2012). On retrouve ces deux caractéristiques dans les documents de référence sur résilience et sécurité alimentaire (ACF International, 2012 ; Béné et al., 2012 ; UE, 2012 ; USAID, 2012).

La lutte contre l'insécurité alimentaire est ainsi redéfinie dans un cadre plurisectoriel tenant compte des interactions homme-environnement. Un plaidoyer émerge alors pour coupler les interventions spécifiques de sécurité alimentaire à d'autres telles que des interventions de prévention et gestion des catastrophes naturelles (DFID, 2011; FAO, 2013; UNFPA, 2015), de réponses au changement climatique (UNSCN, 2010 ; WFP, 2011; ACF International, 2012), prenant en compte les dimensions tant économiques, que politiques, sociales, culturelles et naturelles (Heijmans, 2013).

Les acteurs du développement proposent de considérer la résilience à différentes échelles : individus, ménages, familles, communautés ou encore pays (DFID, 2011 ; USAID, 2012). En témoigne le dernier rapport sur l'état de la population mondiale (UNFPA, 2015) qui considère que « le renforcement de la résilience est un processus cumulatif et progressif à plusieurs niveaux (mondial, régional, national, communautaire et individuel) ».

\section{Les défis de l'opérationnalisation de la résilience dans le domaine de la sécurité alimentaire}

La transposition de la notion de résilience dans le domaine du développement et de la sécurité alimentaire apparaît comme un approfondissement conceptuel, cette notion étant centrée sur la prise en compte des trajectoires longues, et une approche systémique et intégratrice des niveaux d'analyses.

Pour autant, l'opérationnalisation de la résilience dans la sécurité alimentaire se heurte à des visions multiples de sa définition (pas totalement partagée entre des visions plutôt " écologiques » et d'autres plutôt « développement »), et aux problèmes classiques de passage entre une approche théorique systémique et une action où des hiérarchies, des choix doivent être opérés.

La résilience est entendue non pas seulement comme une approche pour analyser des situations et concevoir des actions, mais aussi comme une caractéristique des systèmes et des individus qu'il s'agit d'apprécier, mesurer, suivre, à travers des indicateurs. La difficulté de l'opérationnalisation s'illustre alors quand il s'agit de proposer des indicateurs de suivi et d'évaluation de la résilience. Un bon indicateur doit être « spécifique, mesurable, acceptable, réaliste et temporellement défini («SMART ») et la résilience ne se mesure pas facilement de cette façon. Certains (USAID, 2013) proposent des grilles d'indicateurs ; d'autres (Alinovi et al., 2009 ; FSIN, 2014a ; Gombo Boukary et al., 2016) s'attachent à concevoir un indicateur composite de la résilience. C'est notamment le cas du projet RIMA (Resilience Index Measurement and
Analysis) de la FAO qui cherche à concevoir une méthode quantitative permettant d'expliquer les différences de résistance aux perturbations des ménages, à partir d'un ensemble d'indicateurs rassemblés en quatre piliers : capacité d'adaptation, filets de protection sociale, actifs productifs ou non et accès aux services de base (FAO, 2014 ; d'Erricco, 2015). Enfin, d'autres argumentent en faveur d'un processus d'évaluation qui permet d'analyser la trajectoire du système puis de penser sous forme de scénarios ses futures trajectoires. Tous sont confrontés à la difficulté de prise en compte de toutes les dimensions temporelles et conceptuelles (accessibilité économique, disponibilité, durabilité, sociale, environnementale, etc.) que couvrent la résilience dans le domaine de la sécurité alimentaire sans rendre la mesure trop complexe.

Cette difficulté s'illustre également dans les niveaux/ échelles des interventions et leur articulation. Les outils de mesure de la résilience ont été majoritairement développés pour analyser la résilience au niveau des ménages (Alinovi et al., 2009 ; Alinovi et al., 2010) et permettent d'évaluer des interventions de sécurité alimentaire qui ciblent des individus ou des ménages (CARE France, 2014 ; Gret, 2014 ; GIZ, 2015). On observe en effet, au travers des documents de projets, de programmes ou de politiques, que les interventions qualifiées de renforcement de la résilience correspondent en grande partie à des transferts de biens (intrants par exemple) ou d'argent - dits transferts sociaux - ciblés sur des individus ou des ménages (CARE France, 2014 ; USAID, 2015). Ce type d'action n'est pas nouveau en soi, mais tend seulement depuis quelques années à se développer en Afrique, avec la reproduction de programmes emblématiques mis en œuvre au Brésil (Fome zero) ou en Éthiopie (HLPE, 2012). La prise en compte de la résilience contribue à la montée en puissance des transferts sociaux, car ils peuvent tout à la fois être utilisés de façon ponctuelle pour aider les populations à se remettre d'un choc (phase de récupération) et de façon pérenne au travers de politiques de protection sociale. Les interventions de sécurité alimentaire tendent ainsi à évoluer avec la résilience, d'instruments de politiques agricoles - qui restent largement majoritaires - vers des instruments associés à des politiques de protection sociale.

Si cette approche de transferts sociaux ciblés sur des individus/ménages apparaît pragmatique, elle pose néanmoins des difficultés méthodologiques en matière de ciblage. En outre, elle semble presque aller à l'encontre de la notion de résilience dans l'approche écologique et systémique, et ne résout pas les questions, posées, à l'origine de l'intégration de la notion dans le champ de la sécurité alimentaire. Ainsi, plusieurs acteurs travaillent sur des indicateurs de résilience au niveau des communautés pour sortir d'une approche seulement individuelle et mènent des actions de développement de systèmes communautaires de prévention des crises (CARE France, 2014 ; ACF International, 2015), ou focalisent leurs interventions sur les institutions nationales de développement agricole (GIZ, 2015).

En revanche, les niveaux plus englobants, de type régulation sociale, économique ou politique, ne sont pas traités. À notre connaissance, l'évaluation d'une politique de régulation des prix agricoles, de promotion de l'égalité hommes-femmes, de soutien à la diversité agricole, n'a pas encore été entreprise au regard de la résilience et de la sécurité alimentaire. 
En définitive, la difficulté d'opérationnaliser la résilience en matière de sécurité alimentaire renvoie à la difficulté d'utiliser deux concepts aussi intégrateurs et complexes (résilience et sécurité alimentaire) dans la conception et la mise en œuvre même des projets. Les opérateurs de projet (qu'ils soient gouvernementaux ou des ONG) doivent en outre composer avec de multiples contraintes (financière, technique, humaine, etc.) qui restreignent généralement leur capacité d'expérimentation et d'innovation et les amènent à reproduire les actions pour lesquelles ils ont construit une expérience et une expertise. Comment construire un programme nécessitant d'intervenir sur - ou du moins de suivre - autant de facteurs à des niveaux aussi différents ? On observe des stratégies différentes, avec soit des projets plus centrés sur un, deux, voire trois secteurs tels que l'agriculture, la nutrition ou l'égalité entre les sexes (Gret, 2014 ; AFD, 2015), soit des projets qui essayent de prendre en compte toutes les dimensions. Dans le premier cas, le risque est de ne rien changer par rapport aux habitudes et, dans le second, le risque est la confusion.

\section{Conclusion}

Les interventions et les réflexions sur la sécurité alimentaire ont récemment intégré le concept de résilience, comme en témoigne notre analyse de la littérature. Cette intégration donne l'occasion de s'interroger sur ce qu'est la résilience et son caractère normatif. Alors que pour certains, qui se rattachent à l'analyse de la trajectoire des systèmes, la résilience est une propriété de l'objet étudié et que cette propriété peut, dans certains cas, être jugée indésirable, on perçoit qu'en matière de sécurité alimentaire, la résilience est a priori positive et recherchée. Dans ce second contexte, le concept de résilience aide à repenser conjointement les interventions d'urgence et d'aide au développement pour mieux prendre en compte les dynamiques en présence à plusieurs échelles, les trajectoires longues et les temps courts ainsi que la multiplicité et l'imprévisibilité des chocs que subissent les populations les plus vulnérables. Pour être vraiment opérationnelle, l'intégration de la résilience et de la sécurité alimentaire devrait progresser dans deux directions, qui s'opposeront ou s'enrichiront : d'un côté, la définition et l'usage d'indicateurs pour mesurer la résilience dans le but de rendre compte de l'efficacité des actions de développement visant à améliorer cette résilience, et, de l'autre, la mise au point et l'usage de méthodes d'évaluation des trajectoires de développement pour permettre une analyse prospective.

Remerciements. Ce travail a bénéficié du soutien des projets «Production agricole et sécurité alimentaire en Afrique de l'Ouest " (direction scientifique du Cirad) et REPAM " Améliorer la résilience vis-à-vis de la sécurité alimentaire des populations pauvres dans la boucle du Mouhoun, Burkina Faso » (Gret-UE).

\section{Références}

ACF International. 2012. Améliorer la résilience au changement climatique et à la sécurité alimentaire et nutritionnelle. Document de cadrage. Paris, France : Action contre la faim, 17 p. http://www. actioncontrelafaim.org/fr/content/ameliorer-la-resilience-au-change ment-climatique-et-la-securite-alimentaire-et-0.

ACF International. 2015. Guidelines: ACF resilience analysis and measurement tool. Working document. Paris, France : Action contre la faim, $17 \mathrm{p}$.

AFD. 2015. Maghreb (Maroc et Tunisie). Renforcer la résilience de l'agriculture au changement climatique et sécuriser les revenus agricoles. Paris, France : Agence française de développement, 1 p. http://www.afd.fr/jahia/webdav/site/afd/shared/PORTAILS/SEC TEURS/CLIMAT/fiches-2015/MAGHREB_agriculture.pdf.

Alinovi L, Mane E, Romano D. 2009. Measuring household resilience to food insecurity: application to palestinian households. In: Working paper. EC-FAO Food Security Programme Linking Information and Decision Making to Improve Food Security, 35 p. http://www.fsnnetwork.org/sites/default/files/measuring_hou sehold_resilience_to_food_insecurity.pdf.

Alinovi L, d'Erricco M, Mane E, Romano D. 2010. Livelihoods strategies and household resilience to food security: an empirical analysis to Kenya. In: Paper prepared for the Conference on "Promoting Resilience through Social Protection in Sub-Saharan Africa", organised by the European Report of Development in Dakar, Senegal, 28-30 June, 2010, 52 p. http://erd.eui.eu/media/ BackgroundPapers/Alinovi-Romano-D'Erricco-Mane.pdf.

Ancey V, Daviron B, Pesche D. 2014a. La résilience, maillon entre urgence et développement ou buzzword palliatif à la crise de la pensée ? In : Séminaire sécurité alimentaire. Montpellier : Cirad Moisa, 45 p. http://umr-moisa.cirad.fr/content/download/5665/ 38853/version/1/file/VeroniqueAncey\&al_pr\%C3\%A9senta tion_PPt.pdf.

Ancey V, Daviron B, Pesche D. 2014b. Resilience and development: complement, substitute or stopgap solution. Resilience 2014. Resilience and development: mobilizing for transformation. Montpellier: Cirad Moisa, 2 p. http://resilience2014.sciencesconf. org/25910/document.

Arnauld de Sartre X, Hubert B, Bousquet F. 2015. Introduction. À la recherche des concepts heuristiques sur les relations natures/ sociétés. Nat Sci Soc 23: 154-156. doi: 10.1051/nss/2015028.

BAD. 2015. Remédier à la fragilité et renforcer la résilience en Afrique: stratégie du groupe de la Banque africaine de développement pour 2014-2019. Abidjan : Banque africaine de développement, 61 p. http://www.afdb.org/fileadmin/uploads/afdb/ Documents/Policy-Documents/Rem\%C3\%A9dier \%C3\% A0_la_fragilit $\% \mathrm{C} 3 \% \mathrm{~A} 9$ et renforcer_la_r $\% \mathrm{C} 3 \% \mathrm{~A} 9$ silience_en_A frique_-_Strat $\%$ C3\%A9gie_du_Groupe_de_la_BAD_pour_20142019.pdf.

Barrett CB, Constas MA. 2014. Toward a theory of resilience for international development applications. Proc Natl Acad Sci USA 111: 14625-14630. doi: 10.1073/pnas.1320880111.

Béné C, Wood RG, Newsham A, Davies M. 2012. Resilience: new utopia or new tyranny? Reflection about the potentials and limits of the concept of resilience in relation to vulnerability reduction programmes. In: IDS Working Paper 2012 (405) CSP Working Paper (006). Brighton, UK: Institute of Development Studies and Centre for Social Protection, 61 p. http://doi.wiley.com/10.1111/ j.2040-0209.2012.00405.x.

Berkes F, Folke C. 1998. Linking social and ecological systems for resilience and sustainability. Linking social and ecological systems: management practices and social mechanisms for building resilience. Nat Hazards 41(2): 283-295. doi: 10.1007/s11069-006-9036-7.

CARE France. 2014. Tchad : appui au renforcement de la résilience des ménages affectés par la crise alimentaire l'Est du pays. Paris : CARE France. http://www.carefrance.org/care-actions/program mes-monde/tchad-resilience-crice-alimentaire.htm. 
Crombé X, Jézéquel JH, eds. 2007. Niger 2005. Une catastrophe si naturelle. Paris : Karthala. 296 p.

de Schutter O. 2008. Building resilience: a human rights framework for world food and nutrition security. In: Report of the Special Rapporteur on the Right to Food, Olivier de Schutter, 8 September 2008. $A / H R C / 9 / 23$. A/HRC/9/23. New York: UN Human Rights Council, 43 p. http://www.refworld.org/docid/48cf71dd2.html.

DFID. 2011. Defining disaster resilience: a DFID approach paper. London, UK: Department for International Development-UKaid, 19 p. https:/www.gov.uk/government/uploads/system/uploads/ attachment_data/file/186874/defining-disaster-resilience-approachpaper.pdf.

d'Erricco M. 2015. RIMA II: moving forward the development of the resilience index measurement and analysis model. Rome: FAO, 4 p. http://www.fao.org/3/a-i5298e.pdf.

Fan S, Pandya-Lorch R, Yosef S, ed. 2014. Resilience for food and nutrition security. Washington, DC: International Food Policy Research Institute (IFPRI), $211 \mathrm{p}$. http://www.ifpri.org/sites/ default/files/publications/oc79.pdf\#page $=108$.

FAO. 2006. Building resilience for food security and nutrition in the near East and North Africa. Rome, Italie: FAO-Regional Office for the near East and North Africa - RNE, 2 p. http://www.fao.org/3/a$\mathrm{ml} 1968 \mathrm{e} / \mathrm{ml} 1968 \mathrm{e} 15 . \mathrm{pdf}$.

FAO. 2012. La crise alimentaire et nutritionnelle au Sahel : l'urgence d'appuyer la résilience des populations vulnérables. Cadre stratégique de réponse régionale Burkina Faso, Gambie, Mali, Mauritanie, Niger, Tchad, et Sénégal. Rome : FAO, 5 p. http:// www.fao.org/fileadmin/user_upload/sahel/docs/DocProg\%20FAO $\%$ 20SAHEL $\% 20 \mathrm{FR} \% 20 \mathrm{abr} \% \mathrm{C} 3 \%$ A9g\%C3\%A9.pdf.

FAO. 2013. La résilience des moyens d'existence - Réduction des risques de catastrophe pour la sécurité alimentaire et nutritionnelle. Rome, Italie: Division des urgences et de la réhabilitation Organisations des Nations unies pour l'alimentation et l'agriculture, 105 p. http://www.fao.org/3/a-i3270f.pdf.

FAO. 2014. Resilience Index Measurement and Analysis model. Rome: FAO, 13 p. http://www.fao.org/3/a-i4102e.pdf.

Frankenberger T, Campbell J, Njoka J, Spangler T, Nelson S. 2012a. Building resilience to food security shocks in the Horn of Africa (note). 5 p. http://www.fsa.usda.gov/Internet/FSA_File/2012_fran kenberger_note.pdf.

Frankenberger T, Langworthy M, Spangler T, Nelson S. 2012b. Enhancing resilience to food security shocks. White paper (draft). Washington: FSN Network, 57 p. http://www.fsnnetwork.org/sites/ default/files/revised resilience paper may 28.pdf.

Frantzeskaki N, Tidball K, Elmqvist T. 2014. Tips or traps? Advancing understanding to steer clear of impoverishment traps and tipping points. Montpellier. Resilience 2014. http://resilience2014.sciences conf.org/25099.

FSIN. 2014a. Common analytical model for resilience measurement. Causal framework and methodological options. Technical Series. 2. I. FAO, WFP, EU, USAID Food Security Information Network. Resilience Measurement Technical Working Group, 52 p. http:// www.fsincop.net/fileadmin/user_upload/fsin/docs/resources/FSIN Paper2_WEB_1dic\%20\%28WEB\%29.pdf.

FSIN. 2014b. Resilience measurement principles. Toward an agenda for measurement design. Technical Series. 1. I. FAO, WFP, EU, USAID Food Security Information Network. Resilience Measurement Technical Working Group, 31 p. http://www.fao.org/filead min/user_upload/drought/docs/FSIN\%20Resilience\%20Measure ment $\% 20201401$.pdf.

GIZ. 2015. Sécurité alimentaire et renforcement de la résilience. Initiative spéciale «Un seul monde sans faim ». Bonn/Eschborn : Ministère fédéral allemand de la coopération économique et du développement (BMZ), 2 p. https://www.giz.de/en/downloads/ giz2015-fr-seule-monde-sans-faim-securite-alimentaire.pdf.

Gombo Boukary A, Diaw A, Wünscher T. 2016. Factors affecting rural households' resilience to food insecurity in Niger. Sustainability 8: 181. doi: 10.3390/su8030181.

Gret. 2014. Améliorer la résilience des populations vulnérables en matière de sécurité alimentaire et nutritionnelle. In : Projet Résanut. Nogent-sur-Marne, France, 4 p. http://www.gret.org/wp-content/ uploads/FICHE PROJET RESANUT-basse-d\%C3\%A9f.pdf.

Haider J, Wijermans N, Peterson G, Schl M. 2014. Perverse resilience: a systematic review of traps in social-ecological systems (abstract). Montpellier: Resilience 2014, 2 p. http://resilience2014.sciences conf.org/25088.

Heijmans A. 2013. Manuel résilience 2.0 pour les acteurs de l'aide et les décideurs dans les domaines de la réduction des risques de catastrophe, de l'adaptation au changement climatique et de la réduction de la pauvreté. Wageningen : Groupe urgence réhabilitation et développement, Université de Wageningen et Care Hollande, 143 p. http://www.reachingresilience.org/IMG/pdf/resilience-hand book-fr.pdf.

Hillier D, Castillo GE. 2013. Pas de hasard : résilience et inégalités face au risque. Oxford, UK : Oxfam GB pour Oxfam International, 37 p. http://policy-practice.oxfam.org.uk/publications/no-accidentresilience-and-the-inequality-of-risk-292353.

HLPE. 2012. La protection sociale pour la sécurité alimentaire. In : Rapport du groupe d'experts de haut niveau sur la sécurité alimentaire et la nutrition du Comité de la sécurité alimentaire mondiale, Rome, 2012. http://www.fao.org/3/a-me422f.pdf.

Holling CS. 1973. Resilience and stability of ecological systems. Annu Rev Ecol Syst 4: 1-24. doi: 22160726.

Holling CS. 1996. Engineering resilience versus ecological resilience. In: National Academy of Engineering, ed. Engineering within ecological constraints. Washington DC (USA): The National Academies Press, pp. 31-44. http://www.nap.edu/read/4919/chap ter/4.

Janin P, Dury S. 2012. Les nouvelles frontières de la sécurité alimentaire. Une approche prospective. Cah Agric 21: 285-292. doi: 10.1684/agr.2012.0585.

Lallau B. 2011. La résilience, moyen et fin d'un développement durable ? Ethique Econ 8: 168-185. doi: 10.4000/developpement durable.1143.

Lallau B. 2014. La résilience contre la faim - Nouvelle donne ou nouvel artifice ? In : Journées de recherches en sciences sociales, SFER INRA Cirad. Lille, France, 22 p. http://resiliences.univ-lille1. fr/wp-content/uploads/LALLAU-SFER-d\%C3\%A9cembre-2014. pdf.

MAE. 2014. La résilience dans le cadre de la sécurité alimentaire. Paris, France : Ministère des Affaires étrangères et du Développement international. http://www.diplomatie.gouv.fr/fr/politiqueetrangere-de-la-france/sante-securite-alimentaire-education-etgenre/securite-alimentaire/article/la-resilience-dans-le-cadre-de-la.

Mathevet R, Bousquet F. 2014. Résilience et environnement : penser les changements socio-écologiques. Paris (France): BuchetChastel Éditions, 176 p.

Maxwell S, Smith M. 1992. Household food security: a conceptual review. In: Maxwell S, Frankenberger T, eds. Household food security: concepts, indicators, and measurements: a technical review. New York (USA) and Rome (Italie): UNICEF and IFAD, pp. 1-72. https://www.ifad.org/documents/10180/ 4b29b799-49a9-4740-bfab-4bbd24059b56.

Michiels D, Egg J. 2007. Les politiques de prévention et gestion des crises alimentaires. Enseignements de la crise du Niger en 2005. Paris : Direction générale de la Coopération internationale et du 
développement, 90 p. http://www.diplomatie.gouv.fr/fr/IMG/pdf/ Rapport_Niger.pdf.

Michiels D, Egg J, Blein R. 2012. La répétition des crises alimentaires et nutritionnelles au Niger : la rénovation urgente des politiques de sécurité alimentaire. Cah Agric 21: 302-310. doi: 10.1684/ agr.2012.0588.

Phelan L, Henderson-Sellers A, Taplin R. 2013. The political economy of addressing the climate crisis in the earth system: undermining perverse resilience. $N$ Polit Econ 18: 198-226. doi: 10.1080/ 13563467.2012.678820.

Pingali P, Alinovi L, Sutton J. 2005. Food security in complex emergencies: enhancing food system resilience. Disasters 29: 5-24. doi: 10.1111/j. 0361-3666. 2005.00282.x.

Quenault B. 2015. De Hyōgo à Sendai, la résilience comme impératif d'adaptation aux risques de catastrophe : nouvelle valeur universelle ou gouvernement par la catastrophe ? Dév Durable Territ 6(3). doi: 10.4000/developpementdurable.11010.

Resilience Alliance. 2010. Assessing resilience in social-ecological systems: workbook for practitioners. Version 2.0, $53 \mathrm{p}$. http://www. resalliance.org/files/ResilienceAssessmentV2_2.pdf.

Save the Children. 2015. Non-emergency food security and livelihoods. site web. Fairfield, CT, USA: Save the Children, 1 p. http://www.savethechildren.org/site/c.8rKLIXMGIpI4E/ b.9316579/k.9DA/NonEmergency_Food_Security_and_Liveli hoods.htm.

Touzard JM, Temple L. 2012. Sécurisation alimentaire et innovations dans l'agriculture et l'agroalimentaire : vers un nouvel agenda de recherche ? Une revue de la littérature. Cah Agric 21: 293-301. doi: 10.1684/agr.2012.0577.

UE. 2001. Linking relief, rehabilitation and development - An assessment. Communication from the Commission to the Council and the European Parliament. 153. Brussels: European Commission, 23 p. http://eur-lex.europa.eu/LexUriServ/LexUriServ.do? uri=COM:2001:0153:FIN:EN:PDF.

UE. 2012. The EU approach to resilience, learning from food security crisis. $\operatorname{COM}(2012) 586$ final. Brussels, Belgium: European Commission, 13 p. http://ec.europa.eu/echo/files/policies/resi lience/com_2012_586_resilience_en.pdf.

UNFPA. 2015. State of the world population 2015. Shelter from the storm. A transformative agenda for women and girls in a crisisprone world. New York (USA): The United Nations Population Fund, 136 p. https:/www.unfpa.org/sites/default/files/sowp/down loads/State_of_World_Population_2015_EN.pdf.

UNSCN. $201 \overline{0}$. Climate change and nutrition security. Message to the UNFCCC negociators. In: United Nations Framework Convention on Climate Change - 16th United Nations Conference of the Parties (COP16). Cancun, Mexique: United Nations System Standing Committee on Nutrition, 11 p. http://www.unscn.org/files/State ments/Bdef_NutCC_2311_final.pdf.

USAID. 2012. Building resilience to recurrent crisis - USAID policy and program guidance. Washington DC, USA: U.S. Agency for International Development, $27 \mathrm{p}$. https://www.usaid.gov/sites/ default/files/documents/1870/USAIDResiliencePolicyGuidanceDo cument.pdf.

USAID. 2013. The resilience agenda: measuring resilience in USAID. Washington DC, USA: U.S. Agency for International Development, 7 p. https://www.usaid.gov/sites/default/files/documents/1866/ Technical $\% 20$ Note_Measuring\%20Resilience $\% 20$ in $\% 20$ USAID_ June \%202013.pdf.

USAID. 2015. USAID food for peace activity supports food security and drought resilience in rural communities. Available from https:// www.usaid.gov/zimbabwe/news-information/press-releases/usaidfood-peace-activity-supports-food-security-and (last consult: 2016/ 14/06).

VOICE, CONCORD. 2012. Document de principe. Associer l'aide d'urgence, la réhabilitation et le développement (LRRD) : vers une approche mieux concertée pour améliorer la résilience et l'impact. 6 p. http://www.ngovoice.org/documents/VOICE-CONCORD\% 20LRRD $\% 20$ Position \%20paper\%20FR\%20FINAL \%20formated. pdf.

Vonthron S. 2014. Caractérisation de la résilience des exploitations agricoles face à l'insécurité alimentaire : diagnostic agro-socioéconomique pour le projet REPAM dans la boucle du Mouhoun. Montpellier : SupAgro, 147 p. https://web.supagro.inra.fr/pmb/ opac_css/index.php?lvl=author_see\&id=70481.

Vonthron S, Alpha A, Bousquet F, Dury S, Fallot A. 2015. Résilience et sécurité alimentaire et nutritionnelle : concepts et évaluation. In : Rapport scientifique du bilan thématique programmé " Production agricole et sécurité alimentaire en Afrique de l'Ouest». Montpellier : Cirad/Métaprogramme GloFoodS, 51 p.

Walker B, Holling CS, Carpenter SR, Kinzig A. 2004. Resilience, adaptability and transformability in social-ecological systems. Ecol Soc V 9: 5. doi: 18129228.

WFP. 2011. Climate change and hunger: towards a WFP policy on climate change. Informal consultation. C-10367E. Rome, Italie: World Food Programme, 15 p. http://documents.wfp.org/stellent/ groups/public/documents/resources/wfp232740.pdf.

WFP. 2013. Building resilience through asset creation. Rome, Italie: World Food Programme, 20 p. http://documents.wfp.org/stellent/ groups/public/documents/communications/wfp261744.pdf.

Citation de l'article : Vonthron S, Dury S, Fallot A, Alpha A, Bousquet F. 2016. L'intégration des concepts de résilience dans le domaine de la sécurité alimentaire : regards croisés. Cah. Agric. 25: 64001. 